\title{
Stage of Charge Estimation of Lithium-ion Battery Packs Based on Improved Cubature Kalman Filter with Long Short-Term Memory Model
}

\author{
Xing Shu ${ }^{1}$, Guang $\mathrm{Li}^{2}$, Yuanjian Zhang ${ }^{3}$, Shiquan Shen ${ }^{1}$, Zheng Chen ${ }^{1,2^{*}}$ and Yonggang Liu ${ }^{4 *}$ \\ ${ }^{1}$ Faculty of Transportation Engineering, Kunming University of Science and Technology, Kunming, 650500, China \\ ${ }^{2}$ School of Engineering and Materials Science, Queen Mary University of London, London, E1 4NS, United \\ Kingdom \\ ${ }^{3}$ School of Mechanical and Aerospace Engineering, Queen's University of Belfast, BT9 5AG, Northern Ireland. \\ ${ }^{4}$ State Key Laboratory of Mechanical Transmissions \& School of Automotive Engineering, Chongqing University, \\ Chongqing, 400044, China \\ Email: shuxing92@kust.edu.cn, g.li@qmul.ac.uk,y.zhang@qub.ac.uk, shiquan@tju.edu.cn, chen@kust.edu.cn, \\ andylyg@umich.edu \\ Corresponding Author: Zheng Chen (chen@kust.edu.cn, +86 13008659331) and Yonggang Liu \\ (andylyg@umich.edu, +86 18696650900)
}

\begin{abstract}
Accurate estimation of state of charge (SOC) of lithium-ion battery packs remains challenging due to inconsistencies among battery cells. To achieve precise SOC estimation of battery packs, firstly, a long short-term memory (LSTM) recurrent neural network (RNN)-based model is constructed to characterize the battery electrical performance, and a rolling learning method is proposed to update the model parameters for improving the model accuracy. Then, an improved square root-cubature Kalman filter (SRCKF) is designed together with the multi-innovation technique to estimate battery cell's SOC. Next, to cope with inconsistencies among battery cells, the SOC estimation value from the maximum and minimum cells are combined with a smoothing method to estimate the pack SOC. The robustness and accuracy of the proposed battery model and cell SOC estimation method are verified by exerting the experimental validation under time-varying temperature conditions. Finally, real operation data are collected from an electric-scooter (ES) monitoring platform to further validate the generalization of the designed pack SOC estimation algorithm. The experimental results manifest that the SOC estimation error can be limited within $2 \%$ after convergence.
\end{abstract}

Key Words: Inconsistency, long short-term memory recurrent neural network, square root cubature Kalman filter, state of charge. 


\section{INTRODUCTION}

Transportation electrification represents a promising solution to mitigate greenhouse gas (GHG) emission and environmental pollution; and this provides favorable opportunities for prompting development of electric vehicles (EVs) and electric-scooters (ESs) [1]. Lithium-ion batteries have been dominating the energy storage media of EVs and ESs [2], of which the energy storage systems are usually composed of hundreds to thousands of cells connected in specific topologies [3]. This leads to the increasing demand of designing effective high-performance battery management systems (BMS), of which one critical task is to conduct state of charge (SOC) estimation of batteries. Accurate knowledge of SOC can not only contribute to remaining driving range estimation and proper charge/discharge operations, but also facilitate batteries to operate in a safer range [4]. SOC cannot be measured directly, but can be estimated/induced from external measurements such as current, voltage and temperature. Due to heterogeneous operation conditions (such as wide time-varying temperature range), dynamic and highly nonlinear characteristics (different load and aging status) of lithium-ion batteries, it is still an intractable task to achieve accurate estimation of SOC with full operation range and lifespan [5].

Nowadays, a variety of intelligent methods have progressively merged for SOC prediction of cells or battery packs. They can be generally divided into four categories: ampere-hour (Ah) integration method, open circuit voltage (OCV)-based method, data driven methods and filter-based methods [6]. Among them, the Ah integration method is usually exerted, in combination with the OCV-based method, to incorporate their individual advantages for achieving fast reliable estimation [7]. Although it is simple and easy to implement, the method shows poor estimation accuracy due to the open-loop control without difference correction [8]. Data driven methods, such as neural networks (NNs), supported vector machine (SVM), can excavate the hidden nonlinear relationship between external feature variables and SOC variation through large amounts of experimental data [9]. However, data driven methods require a large amount of training data to delineate the hidden nonlinear behaviors of lithium-ion batteries for predicting SOC. Moreover, it is not realistic to obtain the SOC with satisfactory accuracy in real operation. 
The filter-based methods are the mainstream solutions for SOC estimation. The widely used solutions include Kalman filter (KF), proportional-integral (PI) observer [10], H-infinite filter (HIF) [11], particle filter (PF) [12] as well as their extensions [13]. As well known, extended Kalman filter (EKF) is a classical observation algorithm for SOC estimation of lithium-ion batteries, and it mainly employs the partial derivatives to linearize the nonlinear electrical characteristics of batteries [14]. Nevertheless, the noise covariance of process and measurement are assumed to be constant values, thus reducing the estimation precision and incurring error divergence. The adaptive covariance calculation based EKF (AEKF) is introduced to mitigate the error divergence by iteratively updating the noise covariance matrices [15]. However, a common limitation of AEKF and EKF algorithms is that require to calculate the so-called Jacobian matrix during estimation, thus contradicting the accuracy of diagnostic SOC. To lessen the computational burden of solving the Jacobian matrix and diminish the linearization error, the cubature KF (CKF) method is proposed on the basis of the third-degree spherical-radial cubature law [16]. It exploits a group of $2 n$ sampling points to calculate the covariance and mean value of the state. In addition to the accuracy improvement, the CKF, compared to EKF and AEKF, features better computational efficiency and stability, [17]. However, it also involves some imperfections. One obvious defect is that solving the Cholesky decomposition of the positive semi-definite matrix during estimation may easily result in divergence.

For these SOC estimation algorithms, inaccurate mathematical model can generate a non-negligible passive influence on SOC estimation. Generally, battery models can be simply sorted into three types: white-box models, grey-box models and black-box models [18], which mainly differ in modeling construction, computational efficiency, simulation precision and modeling output results. White-box models refer to the models which are based on complex electrochemical mechanism, and a set of partial differential equations (PDEs) are usually exploited to describe the physical and electrochemical dynamics inside of lithium-ion batteries [19]. However, resolving a number of PDEs will undoubtedly increase the computational burden. Grey-box models are the most widely adopted in on-board BMS applications due to their fast computation speed, acceptable accuracy and easy interpretability [20]. One representative grey- 
box model is the equivalent circuit model (ECM), which is constituted by a number of equivalent electric components (such as capacitance and resistance), together with OCV sources, to characterize the battery's external electrical performances. However, the model parameters need to be determined offline in advance or online by different algorithms, such as genetic algorithm (GA) [21], particle swarm optimization (PSO) [22], recursive least square (RLS) [23], and KF [24]. Moreover, the influences of temperature, current as well as SOC on the internal properties are difficult to be taken into account in a unified model, and imprecise parameters may lead to inaccurate modeling performance and also be adverse to SOC estimation. Blackbox models represent electrical performances through a universal nonlinear network supplied by machine learning algorithms without perceiving complex electrochemical reactions inside of batteries [25]. Nowadays, with the development of vehicle-to-vehicle (V2V), vehicle-to-cloud (V2C) communication techniques, a large amount of operational data of batteries can be remotely recorded in central monitoring platforms [26]. In this context, the emerging cloud-terminal battery monitoring system can supply a potential manner to mitigate the calculation burden imposed on on-board BMSs and further improve the modeling performance. Moreover, with the accumulation of battery data and the improvement of computing capacity furnished by the graphics-processing unit (GPU), machine learning methods have progressively drawn wide attention. Among the black-box models, long-short term memory (LSTM) network, as an extended formation of recurrent neural network (RNN), can capture the long-term relationship of historical information [27]. Given the characteristics of long-term responses of battery voltage, LSTM network can be adopted as an ideal candidate to model the electrical performances of batteries.

Since battery inconsistency is inevitable in production and operation, the approaches discussed above cannot be directly applied in battery packs consisting of a massive amount of cells [28]. Obviously, it is impertinent to treat a pack as a simple cell when estimating the pack SOC. To now, the widely applied approach for pack SOC estimation is the mean-plus-difference model, which adopts a mean model to identify the mean condition of battery pack and a difference model to assess the diversity of cell SOC and internal resistance [29]. In [30], the second-order ECM and simple resistance model are respectively established as the cell mean model and difference model, and then the EKF is introduced to estimate the 
mean and difference SOC. However, this approach needs to build two battery models, and the number of parameters waiting to be identified also increase. Thus, it is imperative to investigate a simple, reliable and effective pack SOC estimation method to fill in the gaps of existing research.

Motivated by these issues, this study develops an advanced battery pack SOC prediction method considering inconsistence among battery cells. The method combines the improved square root CKF (SRCKF) technique with the LSTM network model to furnish effective cell SOC estimation. Then, the pack SOC is estimated by a smooth weighing method based on the maximum and minimum SOC of battery cells. The validation results based on the laboratory test data and real operation data manifest that the proposed approach can track the variation of reference SOC after quick convergence. Three original contributions of this study are briefly summarized as follows: 1) A fusion method combining the LSTM network and the improved SRCKF algorithm is developed to effectively model the battery's electrical characteristics and estimate the cell SOC. 2) A smoothness weighing method is presented to estimate the pack SOC considering the inconsistency among cells. By taking the maximum and minimum cell SOC values as the features of battery pack, the iteration rules of weights and bias are designed to enhance the estimation precision and inconsistency flexibility of pack SOC. 3) The accuracy and reliability of the proposed battery modeling method and SOC prediction algorithm are justified by full comparison with conventional estimation methods and validated by real operation data.

\section{BATTERY MODEL CONSTRUCTION}

\section{A. Battery Modeling Based on LSTM}

In this section, the LSTM network is employed to simulate the electrical characteristics of batteries. Fig. 1 illustrates the schematic diagram of a general LSTM network, where tanh denotes the hyperbolic function, $\sigma$ is the sigmoid activation function, $\operatorname{In} P_{t}$ and $O u t P_{t-1}$ respectively indicate the input data at current step $t$ and output value at previous step $t-1$ [31]. Different from traditional RNN, LSTM includes three gates, i.e., input, output and forget gates, to attain long-term dependencies. They are merged 
together to determine whether a cell should remember or forget newly obtained information. More details about the LSTM can be referred to our earlier study in [27].

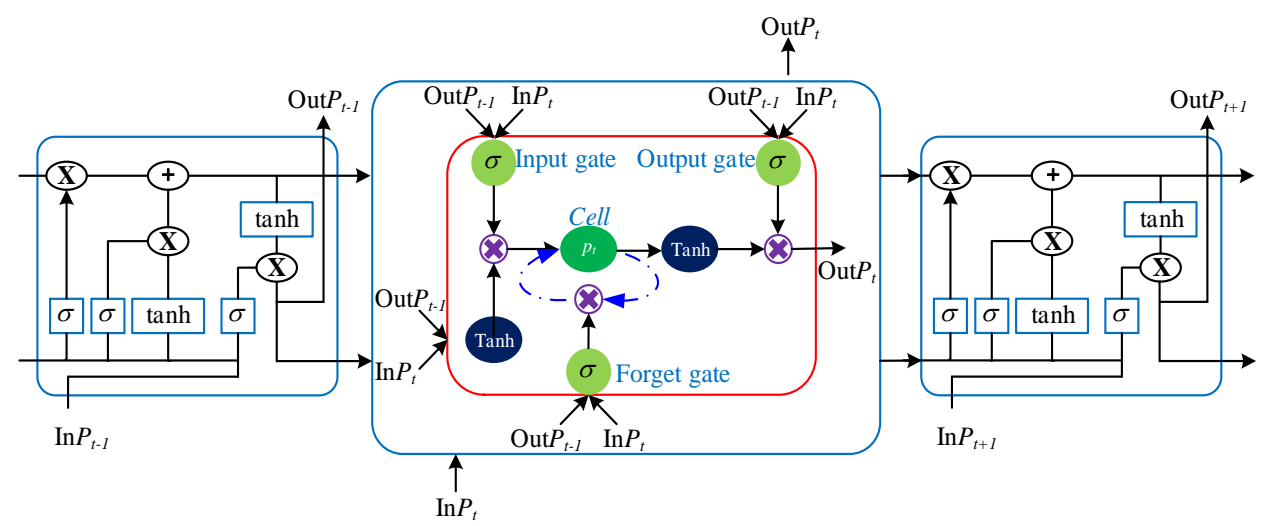

Fig. 1. The network schematic of LSTM network.

Different from ECMs, which need to identify the well-suited model parameters, the LSTM network can directly map the nonlinear relationship between the input and output of a model. This contributes to the process simplification of identifying the model parameters and the independence on specific test for model identification. In the LSTM model, the current $I_{k}$, SOC $s_{k}$, surface temperature $T_{k}$ at step $k$ and the voltage $v_{k-1}$ at step $k-1$ are considered as the inputs of LSTM, represented as $\operatorname{In} P_{k}=\left[I_{k}, s_{k}, T_{k}, v_{k-1}\right]$, and the output of LSTM is the voltage value $v_{k}$ at current step $k$, i.e., $\operatorname{OutP}_{k}=\left[v_{k}\right]$. The SOC is calculated by the Ah integration method, as:

$$
s_{k+1}=s_{k}+\eta I_{k} \Delta t / Q_{n}
$$

where $\eta$ indicates the coulomb efficiency, $\Delta t$ stands for the sampling interval with the unit of second, and $Q_{n}$ denotes the battery rated capacity with the unit of Ah. Here, the root-mean-square error (RMSE) is employed to evaluate the difference between the model output and real test data, as:

$$
R M S E=\sqrt{\frac{1}{N} \sum_{k=1}^{N}\left(v_{k}-v_{k}^{*}\right)^{2}}
$$

where $v_{k}^{*}$ is the ground-truth value of terminal voltage at time step $k$, and $N$ is the number of time steps. Note that 21700 lithium-ion batteries are used in the case study of this paper, of which the main 
parameters are tabulated in Table I. Note that $I$ is negative for discharging and positive for charging. As well known, battery temperature exhibits distinct influence on available capacity. For the batteries under investigation, the initial available capacities at different temperatures are plotted in Fig. 2. It can be clearly found the maximum discharge capacity increases nonlinearly with temperature. Here, a double exponential function is adopted to simulate the correlation:

$$
Q_{n}=q_{1} \exp \left(q_{2} \cdot T\right)+q_{3} \exp \left(q_{4} \cdot T\right)
$$

where $q_{j}, j=1,2,3,4$ are the fitting coefficients.

TABLE I. SPECIFICATIONS OF THE TARGET BATTERY.

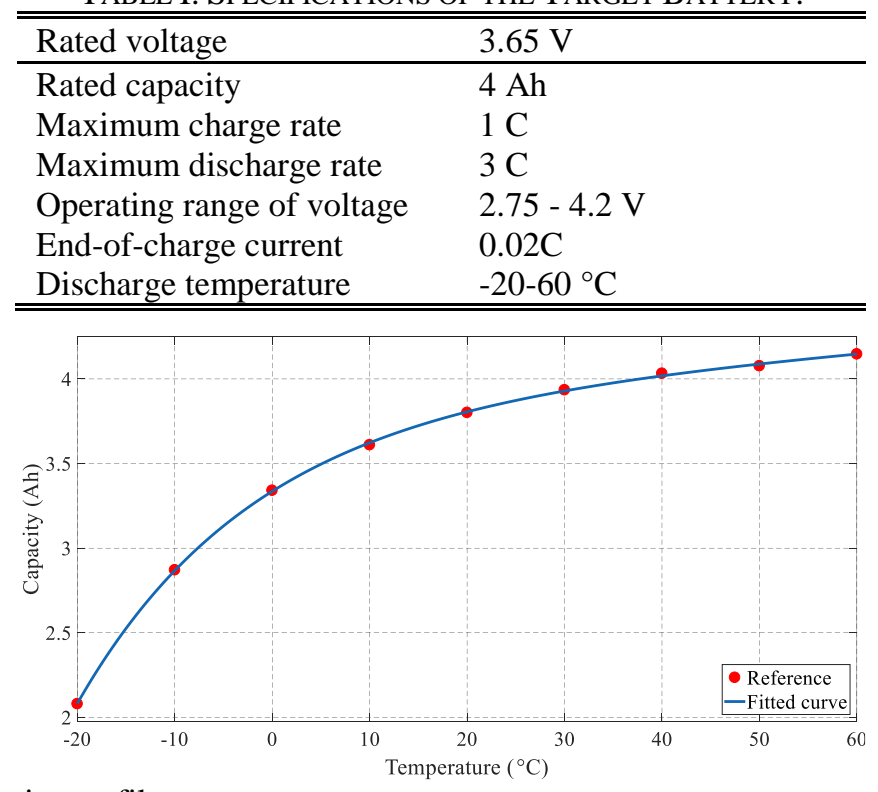

Fig. 2. Available capacity profile.

\section{B. Rolling Learning Method}

The battery model parameters vary with operation throughout their lifetime and are influenced by cycles and ambient temperature. It is difficult to rely on a fixed LSTM model to accommodate external and internal environmental variations through simple offline trainings. Moreover, it is time-consuming when conducting offline tests. To cope with this limitation, a rolling learning method is proposed to continuously learn and identify model parameters, as illustrated in Fig. 3. 


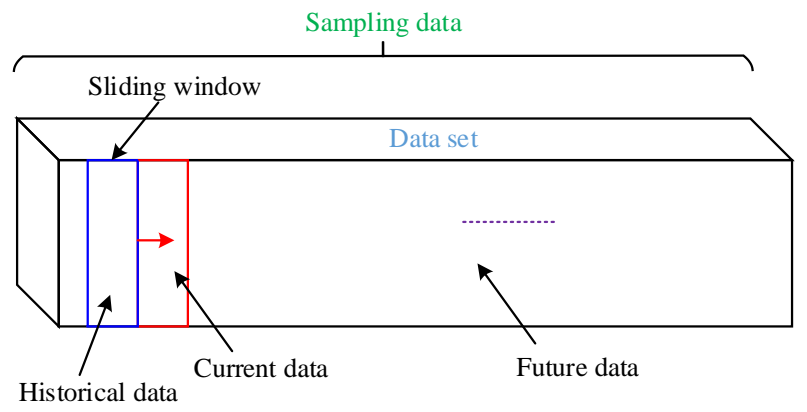

Fig. 3. Illustration of rolling learning method.

As can be seen, the operation data can be split into three categories: historical data, current data and future data. The historical data gradually increase with the accumulation. To avoid the continuously increasing amount of data, a receding horizon $M$ is introduced to store the most recent historical data. The LSTM network will re-train the battery model when the amount of accumulation data is more than the preset $M$ and will exploit the new parameters of LSTM to predict the terminal voltage. With the continuously updated data, the updated model will incorporate the current and the most recent factors for renewing model parameters. Additionally, in practical applications, the initial LSTM model is firstly trained by the test data collected from laboratory. When the LSTM is applied online, the battery operation data will be continuously collected by the central monitoring platform, and then the rolling learning method is employed to learn and update the model parameters under different working conditions.

After the battery model is constructed by the LSTM network, the SOC estimation will be conducted based on reliable simulation of battery electrical characteristics.

\section{StATE OF Charge Estimation Algorithm Design}

This section will firstly introduce the application of improved SRCKF for cell SOC estimation based on the proposed LSTM model, and then a novel SOC inconsistency estimation algorithm is proposed to calibrate the SOC of battery pack.

\section{A. Cell SOC Estimation}

For a discrete nonlinear system, the state-space model subject to noises can be formulated as:

$$
\left\{\begin{array}{l}
x_{k+1}=f\left(x_{k}, u_{k}\right)+w_{k} \\
y_{k}=g\left(x_{k}, u_{k}\right)+h_{k}
\end{array}\right.
$$


where $x_{k}$ and $y_{k}$ denote the state vector and output value at time step $k ; f(\cdot)$ and $g(\cdot)$ indicate the state equation and output equation, respectively. $w_{k}$ and $h_{k}$ are the noises of process and measurement, $u_{k}$ defines as input vector, i.e., current. Normally, the recursive equation of Ah integration method is regarded as the state equation of batteries, as show in (1). The output equation can be represented by the assembled LSTM network, as:

$$
y_{k}=\operatorname{LSTM}\left(I_{k}, s_{k}, T_{k}, v_{k-1}\right)
$$

To overcome the difficulty of calculating the Jacobian matrix of the LSTM model, an improved SRCKF algorithm is introduced to estimate the SOC in this study. For a traditional SRCKF, single information is utilized to update the state estimation, while the multi-innovation approach exploits historical information to readdress the current state, so as to improve the estimation accuracy [22]. The historical information vector of state estimation can be expressed as:

$$
E_{p, k}=\left[\begin{array}{c}
e_{k} \\
e_{k-1} \\
\vdots \\
e_{k-p+1}
\end{array}\right]=\left[\begin{array}{c}
y_{k}^{*}-y\left(\hat{x}_{k}^{-}, u_{k}\right) \\
y_{k-1}^{*}-y\left(\hat{x}_{k-1}^{-}, u_{k-1}\right) \\
\vdots \\
y_{k-p+1}^{*}-y\left(\hat{x}_{k-p+1}^{-}, u_{k-p+1}\right)
\end{array}\right]
$$

where $p$ indicates the historical length, $e_{k}$ is the error matrix at time step $k, y_{k}^{*}$ and $y(\cdot)$ denote the measured and predicted voltages at step $k$, respectively. $\hat{x}_{k}^{-}$represents the priori estimation. Correspondingly, the Kalman gain matrix can be constructed, as:

$$
K_{p, k}=\left[K_{k}, K_{k-1}, \cdots, K_{k-p+1}\right]
$$

The state update equation can be formulated, as:

$$
\hat{x}_{k}^{+}=\hat{x}_{k}^{-}+K_{p, k} E_{p, k}=\hat{x}_{k}^{-}+\sum_{i=1}^{p} K_{k-i+1} e_{k-i+1}
$$

Here, a weight factor is imported in the state update equation to penalize different historical information, as: 


$$
\hat{x}_{k}^{+}=\hat{x}_{k}^{-}+K_{p, k} E_{p, k}=\hat{x}_{k}^{-}+\sum_{i=1}^{p} \lambda^{i} K_{i, k} e_{k-i+1}
$$

where $\lambda$ denotes the weight factor, $\lambda^{i}$ is the penalty coefficient at time step $k-i+1$. To trade-off the calculation burden and estimation accuracy, the historical length $p$ and the weight factor $\lambda$ should be carefully determined. Larger $p$ means that the BMS needs to store more historical data, thus increasing the calculation burden. By contrast, smaller $p$ indicates that a small amount of historical innovation will be exploited to update the current information. Through optimization, $p$ and $\lambda$ are respectively set to 15 and 0.5 by balancing the calculation burden and estimation accuracy. By summarizing the above derivations, the improved SRCFK algorithm based on LSTM model can be described as follows [32], and the structure of the proposed SOC estimation algorithm based on the LSTM model and improved SRCKF is sketched in Fig. 4.

\section{Step 1. Initialization}

$$
\left\{\begin{array}{l}
S_{0 \mid 0}=\operatorname{chol}\left(E\left\{\left[x_{0}-E\left[x_{0}\right]\right]\left[x_{0}-E\left[x_{0}\right]\right]^{T}\right\}\right) \\
\hat{x}_{0 \mid 0}=E\left[x_{0}\right] \\
\xi_{g}=\sqrt{\frac{m}{2}}[1]_{g}, g=1,2, \cdots, m
\end{array}\right.
$$

where $\operatorname{chol}(\cdot)$ is the Cholesky decomposition, $E[\cdot]$ denotes the expectation, $S_{0 \mid 0}$ means the initial value of square roots of the error covariance matrix and is determined by the Cholesky decomposition, $m=2 n$, and $n$ denotes state dimension.

\section{Step 2. Time update}

The cubature points at step $k-1$ are calculated, as:

$$
x_{g, k-1 \mid k-1}=S_{k-1 \mid k-1} \xi_{g}+\hat{x}_{k-1 \mid k-1}
$$

The propagated cubature points can be generated, as:

$$
x_{g, k \mid k-1}^{*}=f\left(x_{g, k-1 \mid k-1}, u_{k}\right)
$$


The prediction state can be estimated, as:

$$
\hat{x}_{k \mid k-1}=\frac{1}{m} \sum_{g=1}^{m} x_{g, k \mid k-1}^{*}
$$

Then, the square-root of prediction error covariance matrix is deduced, as:

$$
S_{k \mid k-1}=\operatorname{Tria}\left[\chi_{k \mid k-1}^{*}, S_{Q, k-1}\right]
$$

where $\operatorname{Tria}(\cdot)$ denotes the $\mathrm{QR}$ decomposition, and $\chi_{k \mid k-1}^{*}$ and $Q_{k-1}$ are defined, as:

$$
\left\{\begin{array}{l}
S_{Q, k-1}=\operatorname{chol}\left(Q_{k-1}\right) \\
\chi_{k \mid k-1}^{*}=\frac{1}{\sqrt{m}}\left[\begin{array}{lll}
x_{1, k \mid k-1}^{*}-\hat{x}_{k \mid k-1} & \cdots & x_{m, k \mid k-1}^{*}-\hat{x}_{k \mid k-1}
\end{array}\right] \\
Q_{k-1}=S_{Q, k-1} S_{Q, k-1}^{T}
\end{array}\right.
$$

\section{Step 3. Measurement update}

The cubature points are recalculated, as:

$$
x_{g, k \mid k-1}=S_{k \mid k-1} \xi_{g}+\hat{x}_{k \mid k-1}
$$

Then, update the propagated measurement cubature points:

$$
y_{g, k \mid k-1}=\operatorname{LSTM}\left(u_{k}, x_{g, k \mid k-1}, T_{k}, v_{k-1}\right)
$$

and estimate the predicted measurement:

$$
\hat{y}_{k \mid k-1}=\frac{1}{m} \sum_{g=1}^{m} y_{g, k \mid k-1}
$$

The square-root of the innovation covariance matrix can be obtained, as:

$$
S_{y y, k \mid k-1}=\operatorname{Tria}\left[\zeta_{k \mid k-1}, S_{R, k}\right]
$$

where $\zeta_{k \mid k-1}$ is defined, as:

$$
\zeta_{k \mid k-1}=\frac{1}{\sqrt{m}}\left[y_{1, k \mid k-1}-\hat{y}_{k \mid k-1} \cdots y_{m, k \mid k-1}-\hat{y}_{k \mid k-1}\right]
$$

The cross-covariance matrix between measurement and state vector can be calculated, as:

$$
P_{x y, k \mid k-1}=\chi_{k \mid k-1} \zeta_{k \mid k-1}^{T}
$$


where $\chi_{k \mid k-1}=\frac{1}{\sqrt{m}}\left[\begin{array}{lll}x_{1, k \mid k-1}-\hat{x}_{k \mid k-1} & \cdots & x_{m, k \mid k-1}-\hat{x}_{k \mid k-1}\end{array}\right]$. The Kalman gain matrix of the improved SRCFK can be solved, as:

$$
K_{k}=\frac{P_{x y, k \mid k-1} / S_{y y, k \mid k-1}^{T}}{S_{y y, k \mid k-1}}
$$

Thus, the optimal value is calculated in (9), and the corresponding square-root of the error covariance matrix is determined, as:

$$
S_{k \mid k}=\operatorname{Tria}\left[\chi_{k \mid k-1}-K_{k} \zeta_{k \mid k-1}, K_{k} S_{R, k}\right]
$$

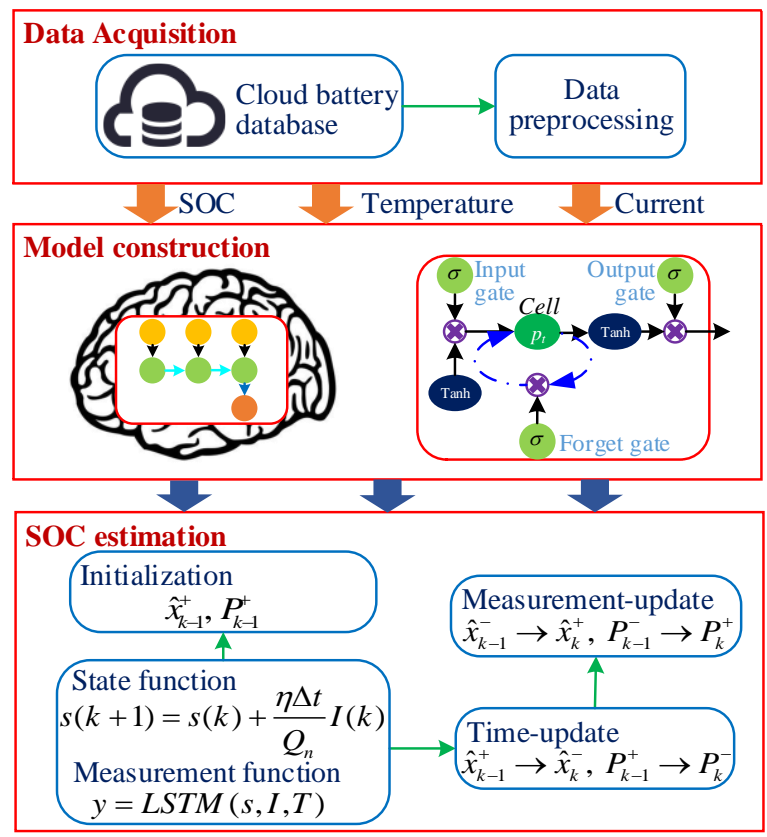

Fig. 4. Structure of SOC estimation algorithm.

\section{B. Pack SOC Estimation}

To guarantee safe operation of battery packs and estimate the pack SOC accurately, a novel smoothness method is proposed for pack SOC estimation by utilizing only the maximum and minimum SOC values of cells. The proposed pack SOC estimation framework is presented in Fig. 5. At each sample, the improved SRCFK algorithm estimates the maximum and minimum SOC of cells, labeled as $s_{\operatorname{Max}}$ and $s_{\text {Min }}$, based on the proposed LSTM model. A common knowledge is that the battery voltage can indirectly reflect the variation trend of battery SOC. Therefore, when selecting the maximum and minimum SOC, we 
only need to compare the voltage of each cell and choose the SOC corresponding to the maximum and minimum voltage. Then, $s_{\text {Max }}$ and $s_{\text {Min }}$ are regarded as the inputs of the smoothness estimation framework, which simultaneously calculate the initial weights $\omega_{1}, \omega_{2}$ and the bias $b$ by:

$$
\left\{\begin{array}{l}
\omega_{1}=\left(s_{\text {Max }}(0)+s_{\text {Min }}(0)\right) / 2 \\
\omega_{2}=1-\omega_{1} \\
b=0
\end{array}\right.
$$

Next, the pack SOC at the initial step can be formulated, as:

$$
s_{\text {Pack }}(0)=\omega_{1} \cdot s_{\text {Max }}(0)+\omega_{2} \cdot s_{\text {Min }}(0)+b
$$

For ideal situations, when the battery is charged, the pack SOC needs to gradually converge to, or equal, the maximum cell SOC, thus preventing overcharge. By contrast, when the battery is discharged, the pack SOC should approach to the minimum SOC of cell, thus avoiding over-discharge. Here we assume that the battery pack is fully discharged after $t_{\text {stop }}$, and the minimum SOC and pack SOC concurrently converge to 0 ; thus, the minimum SOC and pack SOC can be defined, as:

$$
\left\{\begin{array}{l}
s_{\text {Min }}=k_{\text {Min }}\left(t-t_{\text {stop }}\right) \\
s_{\text {Pack }}=k_{\text {Pack }}\left(t-t_{\text {stop }}\right)
\end{array}\right.
$$

where $k_{\text {Min }}$ and $k_{\text {Pack }}$ denote the slopes of the minimum cell SOC and pack SOC, respectively; and $t$ is the running time. In this study, a linear equation is employed to facilitate the SOC estimation of battery pack. During the two adjacent sampling periods, a straight line can be introduced to connect two SOC points. It is assumed that the minimum SOC of the whole process changes linearly with the pack SOC, as:

$$
s_{\text {Pack }}=\omega_{2} \cdot s_{\text {Min }}+b
$$

Thus, we can get: 


$$
\begin{aligned}
& s_{\text {Pack }}=\omega_{2} k_{\text {Min }}\left(t-t_{\text {stop }}\right)+b \\
& \Rightarrow k_{\text {Pack }}\left(t-t_{\text {stop }}\right)=\omega_{2} k_{\text {Min }}\left(t-t_{\text {stop }}\right)+b \\
& \Rightarrow k_{\text {Pack }} t-k_{\text {Pack }} t_{\text {stop }}=\omega_{2} k_{\text {Min }} t-\omega_{2} k_{\text {Min }} t_{\text {stop }}+b \\
& \Rightarrow\left\{\begin{array}{l}
k_{\text {Pack }}=\omega_{2} k_{\text {Min }} \\
k_{\text {Pack }} t_{\text {stop }}=\omega_{2} k_{\text {Min }} t_{\text {stop }}-b
\end{array}\right. \\
& \Rightarrow\left\{\begin{array}{l}
\omega_{2}=\frac{k_{\text {Pack }}}{k_{\text {Min }}} \\
b=\omega_{1} k_{\text {Min }} t_{\text {stop }}-k_{\text {Pack }} t_{\text {stop }} \\
\omega_{2}=\frac{k_{\text {Pack }}}{k_{\text {Min }}}=\frac{\left(k_{\text {Pack }} /\left(t-t_{\text {stop }}\right)\right)}{\left(k_{\text {Min }} /\left(t-t_{\text {stop }}\right)\right)}=\frac{s_{\text {Pack }}}{s_{\text {Min }}} \\
b=\frac{k_{\text {Pack }}}{k_{\text {Min }}} k_{\text {Min }} t_{\text {stop }}-k_{\text {Pack }} t_{\text {stop }}=0
\end{array}\right.
\end{aligned}
$$

The relationship between pack SOC and the minimum SOC can be formulated, as:

$$
s_{P a c k}=\frac{s_{P a c k}}{s_{\text {Min }}} \cdot s_{\text {Min }}
$$

Thus, when the battery is discharged, if the pack SOC is lower than the difference between $s_{\operatorname{Max}}$ and $s_{\text {Min }}$, the weights and bias can be determined by:

$$
\left\{\begin{array}{l}
\omega_{1}=0 \\
\omega_{2}=\frac{s_{P a c k}(t)}{s_{\text {Max }}(t)} \\
b=0
\end{array}\right.
$$

In this case, if the pack SOC is higher than the difference between $s_{\text {Max }}$ and $s_{\text {Min }}$, the weights and bias can be yielded, as:

$$
\left\{\begin{array}{l}
\omega_{1}=\left(s_{\text {Max }}(t)+s_{\text {Min }}(t)\right) / 2 \\
\omega_{2}=1-\omega_{1} \\
b=0
\end{array}\right.
$$

By contrast, when the battery is being charged and the pack SOC is higher than $1-\left(s_{M \text { ax }}-s_{M \text { in }}\right)$, we assume that the battery pack is fully charged after $t_{c h a r g e}$, and the maximum cell SOC and pack SOC concurrently converge to 1 , as: 


$$
\left\{\begin{array}{l}
s_{\text {Max }}=k_{\text {Max }}\left(t-t_{\text {charge }}\right)+1 \\
s_{\text {Pack }}=k_{\text {Pack }}\left(t-t_{\text {charge }}\right)+1
\end{array}\right.
$$

where $k_{M a x}$ indicates the slope of the maximum cell SOC. Similarly, the maximum SOC of the whole process is assumed to change linearly with the pack SOC, as:

$$
s_{\text {Pack }}=\omega_{1} \cdot s_{\text {Max }}+b
$$

Thus, we can attain:

$$
\begin{aligned}
& s_{\text {Pack }}=\omega_{1} \cdot\left(k_{\text {Max }}\left(t-t_{\text {charge }}\right)+1\right)+b \\
& \Rightarrow k_{\text {Pack }}\left(t-t_{\text {charge }}\right)+1=\omega_{1}\left(k_{\text {Max }}\left(t-t_{\text {charge }}\right)+1\right)+b \\
& \Rightarrow k_{\text {Pack }} t-k_{\text {Pack }} t_{\text {charge }}+1=\omega_{1} k_{\text {Max }} t-\omega_{1} k_{\text {Max }} t_{\text {charge }}+\omega_{1}+b \\
& \Rightarrow\left\{\begin{array}{l}
k_{\text {Pack }}=\omega_{1} k_{\text {Max }} \\
1-k_{\text {Pack }} t_{\text {charge }}=\omega_{1}+b-\omega_{1} k_{\text {Max }} t_{\text {charge }}
\end{array}\right. \\
& \Rightarrow\left\{\begin{array}{l}
\omega_{1}=\frac{k_{\text {Pack }}}{k_{\text {Max }}} \\
b=1-k_{\text {Pack }} t_{\text {charge }}+\omega_{1} k_{\text {Max }} t_{\text {charge }}-\omega_{1}
\end{array}\right. \\
& \Rightarrow\left\{\begin{array}{l}
\omega_{1}=\frac{k_{\text {Pack }}}{k_{\text {Max }}}=\frac{\left(s_{\text {Pack }}-1\right) /\left(t-t_{\text {charge }}\right)}{\left(s_{\text {Max }}-1\right) /\left(t-t_{\text {charge }}\right)}=\frac{1-s_{\text {Pack }}}{1-s_{\text {Max }}} \\
b=1-\frac{k_{\text {Pack }}}{k_{\text {Max }}}=1-\omega_{1}
\end{array}\right.
\end{aligned}
$$

The weights and bias can be calculated, as:

$$
\left\{\begin{array}{l}
\omega_{1}=\left(1-s_{\text {Pack }}(t)\right) /\left(1-s_{\text {Max }}(t)\right) \\
\omega_{2}=0 \\
b=1-\omega_{1}
\end{array}\right.
$$

If the pack SOC is lower than $1-\left(s_{\max }-s_{\min }\right)$, the weights and bias can be calculated according to (31).

Eventually, the pack SOC can be determined, as:

$$
s_{\text {Pack }}(t)=\omega_{1} \cdot s_{\text {Max }}(t)+\omega_{2} \cdot s_{\text {Min }}(t)+b
$$

By this manner, the weights and bias can be dynamically adjusted according to $s_{\operatorname{Max}}$ and $s_{\operatorname{Min}}$, thereby enabling the pack SOC to track the maximum or minimum SOC. Note that the pack SOC prediction framework designed in this study can be adaptively regulated to follow the decline rate of cell SOC by 
modifying the bias value. In other words, the bias value can be modified so that the pack SOC is consistent with the limitation of highest or lowest threshold at the specified SOC. Moreover, in the proposed battery pack SOC estimation framework, only the maximum and minimum cell SOC will be employed as the key variables for pack SOC calculation. In short, we only need to calculate the SOC of two cells, and thus the calculation burden of BMS is not high. If there are more battery packs in the EV, we only need to compare the voltage value of each cell and select the cells with the maximum and minimum voltage. Then, the proposed cell SOC estimation method will be employed to estimate the corresponding SOC, followed by the pack SOC estimation based on the smooth weighing method. From this point of view, increasing the number of battery packs will only augment the number of voltage comparisons, and the estimation speed of the battery pack SOC will not change significantly.

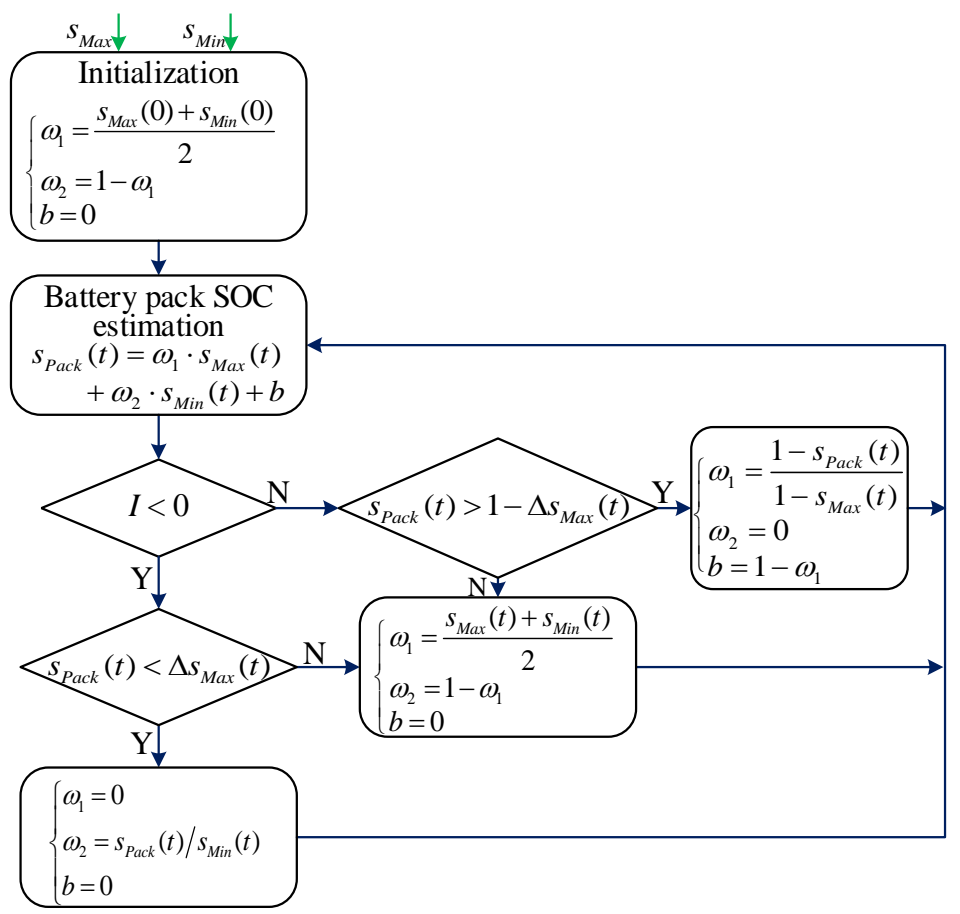

Fig. 5. The flowchart of the battery pack SOC estimation.

In the next step, the SOC estimation will be conducted based on the LSTM model and the proposed algorithm.

\section{Performance Evaluation of Battery Cell}

In this section, the influence of different numbers of units on the precision of LSTM model will be discussed, and then the performance of LSTM model is compared with traditional ECMs. Subsequently, 
the estimation results of the presented cell SOC estimation algorithm are compared with those of typical methods. Finally, the SOC estimation results are assessed under time-varying temperature environment.

\section{A. Parameter Selection}

The model parameters need to be set and optimized before training the LSTM model and estimating the cell SOC. A common knowledge is that different numbers of LSTM units will lead to different modeling accuracy. To determine the proper number, different numbers of hidden units are employed to construct the model. In this experiment, the test is performed at room temperature, i.e., $25{ }^{\circ} \mathrm{C}$. The cell is first fully charged by the constant current-constant voltage (CC-CV) scheme, and then the urban dynamometer driving schedule (UDDS) experiment is cycled until the terminal voltage drops to the cut-off voltage of $2.75 \mathrm{~V}$. The test data are logged with a sampling frequency of $1 \mathrm{~Hz}$. Fig. 6 (a) and (b) show the voltage response results of varying unit numbers of LSTM from 20 to 500, and the interval is set to 20 . To further evaluate the prediction performance of the proposed LSTM model, the model output is compared with the corresponding references. The evaluation criteria, including the average absolute error (AAE), maximum absolute error (MAE), RMSE and running time are considered as the indicators for ease of comparison. The statistic results based on different numbers of units are depicted in Fig. 6 (c) to (d). As can be observed, the minimum AAE, RMSE and running time appear in 20 units. While the optimal MAE of the proposed model is $27 \mathrm{mV}$ and appears when 100 units are selected. From the statistic results, it is difficult to intuitively determine which unit number is the best choice. Here, the entropy weight method is employed to score the prediction results [33]. Assuming that there exist $M I$ evaluation indexes and $N O$ evaluation objects in the original data, the performance value $P_{U n, I n}$ is normalized and limited to the range of $[0,1]$ for minimizing the difference between the data of each dimension of the evaluation index, as:

$$
P_{U n, I n}=\frac{I V_{U n, I n}-\min \left(I V_{\cdot, I n}\right)}{\max \left(I V_{\cdot, I n}\right)-\min \left(I V_{\cdot, I n}\right)}
$$


where $I V_{U n, I n}$ means the $I n$th index value of the $U n$th unit, $\min \left(I V_{\cdot I n}\right)$ and $\max \left(I V_{\cdot I n}\right)$ represent the minimum and maximum value of the Inth index, respectively; $U n \leq M I$; and $I n \leq N O$. Then, the entropy of the Inth index, $E n_{I n}$, can be calculated, as:

$$
E n_{I n}=-(\log N O)^{-1} \sum_{j=I n}^{N O} P_{U n, I n} \log P_{U n, I n}
$$

Each index's entropy weight $w_{I n}$ can be deduced as:

$$
w_{I n}=\frac{1-P_{I n}}{N O-\sum_{I n=1}^{N O} P_{I n}}
$$

Thus, 25 model scores Score $_{I n}$ can be calculated as:

$$
\text { Score }_{I n}=P_{U n, I n} w_{I n}^{T}
$$

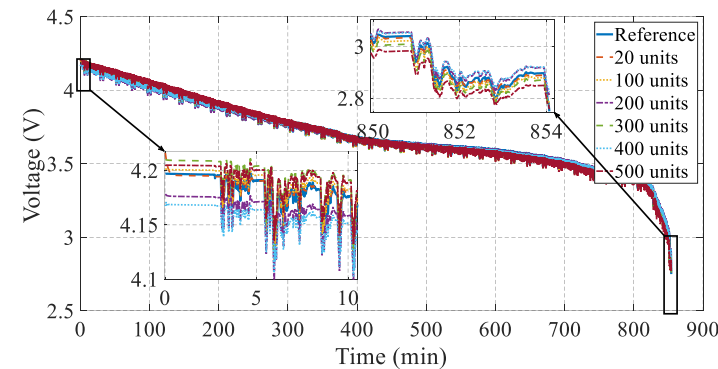

(a)

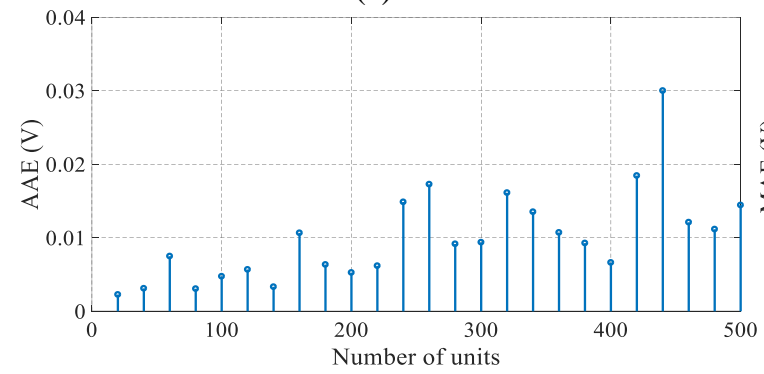

(c)

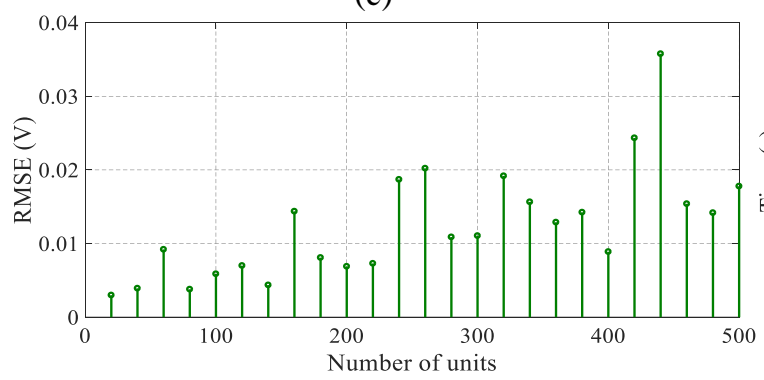

(e)

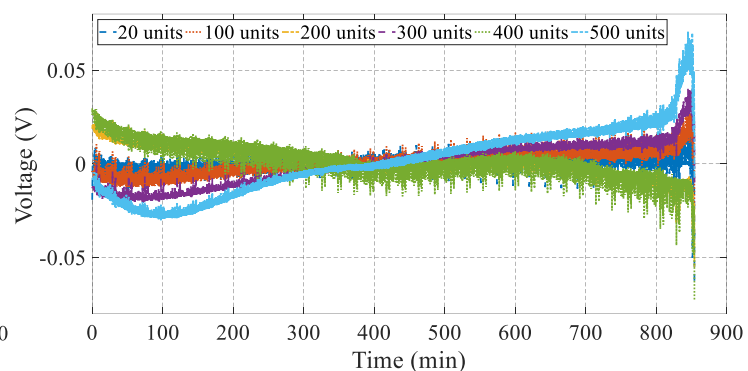

(b)

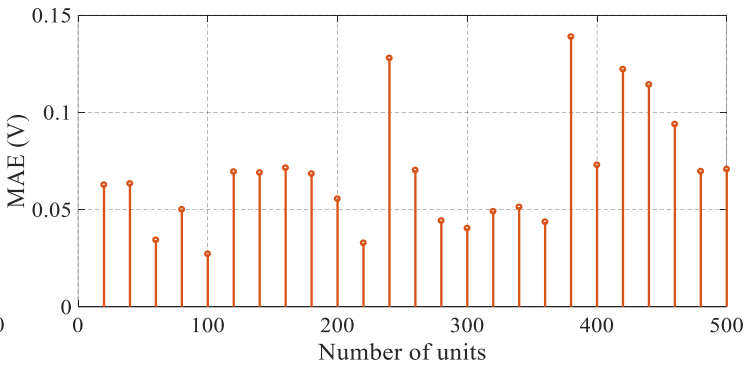

(d)

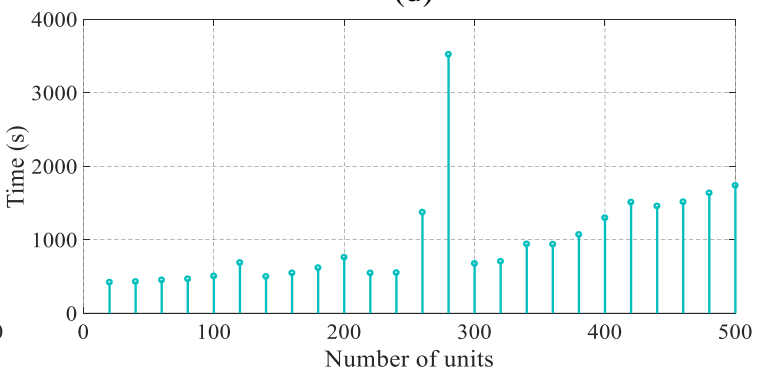

(f)

Fig. 6. Estimation results using different numbers of hidden units. (a) Comparison of voltage. (b) Voltage estimation errors. (c) Voltage AAE. (d) Voltage MAE. (e) Voltage RMSE. (f) Running time. 
By this manner, the scores of 25 models can be calculated, as presented in Fig. 7. It can be discerned that the LSTM model with 100 units features the highest score of 0.95 , while the model with 440 units has the lowest score of 0.3 . This is because when 100 units are selected, the MAE is the least (43\% of that by 20 units and $23 \%$ of that by 440 units). The AAE and RMSE are only $0.79 \mathrm{mV}$ and $0.8 \mathrm{mV}$ less than that by 20 units. Additionally, the running time is merely $80 \mathrm{~s}$ longer than that when 20 units are selected and 952.8 s less than that when 440 units are employed, manifesting that the LSTM model with 100 units can achieve a better trade-off between prediction precision of voltage and running time. Thus, 100 hidden units are suggested for the battery model according to the prediction results in this study.

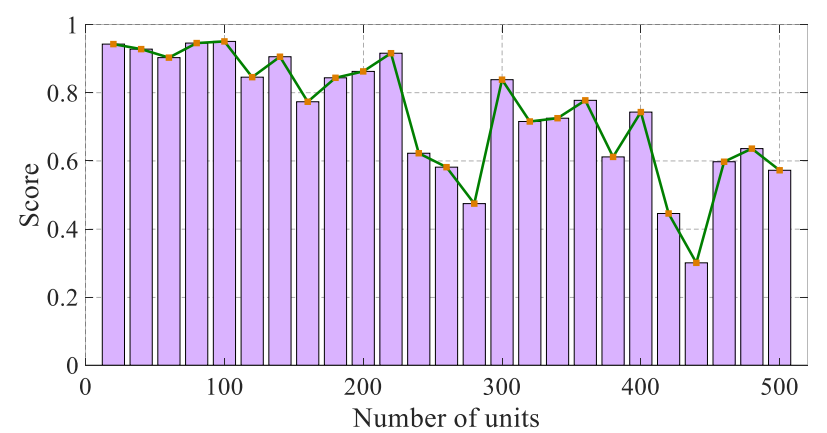

Fig. 7. The scores of different numbers of units.

\section{B. Comparison with Different Battery Models}

As well known, the battery performance highly relies on the operating temperature. With the increase of temperature, the ohmic and polarization resistances during charge and discharge processes decrease gradually, and the reaction rate of the electrode increases, thus promoting the output capacity of battery. In the traditional ECM, these models cannot adapt to the variation of battery temperature due to the static modeling characteristics. To future validate the adaptability of the developed battery model, the voltage prediction performance of different modeling methods is compared at higher temperatures. After obtaining the optimization number of units, the online voltage prediction is performed with the UDDS test at $44{ }^{\circ} \mathrm{C}$. Here, two different ECMs, the first-order and second-order ECMs, with two different parameters identification methods, including GA and PSO, are compared with the recommended modeling method. Moreover, the training data of LSTM are collected from a hybrid test cycle, which contains the UDDS and the federal urban driving schedule (FUDS) test under the temperature range of $-10{ }^{\circ} \mathrm{C}$ to $50{ }^{\circ} \mathrm{C}$ with $10{ }^{\circ} \mathrm{C}$ 
as the increase step. The evaluation results of these three battery models and two identification approaches are demonstrated in Fig. 8, and the statistics results are listed in Table II. We can find that the predicted voltage values based on the LSTM model are always more precise than the other four models. For the ECMs, when the SOC is located in the middle and high range $(15 \%-100 \%)$, the prediction error of the model is generally small; whereas when the SOC drops below $15 \%$, the error gradually increases. The reason is that the polarization at low SOC becomes more obvious, and it is difficult for traditional identification methods to track the rapid change of terminal voltage. In addition, more RC networks cannot significantly improve the performance of model prediction. By comparing with the parameters identified methods based on GA and PSO, it can be clearly found that the performance of PSO is slightly better than that of GA.

By the proposed model, the MAEs have been well confined within $30 \mathrm{mV}$, occupying about $0.82 \%$ of nominal voltage. However, the least MAE of other four traditional models is higher than $260 \mathrm{mV}$, which is about nine times larger than that of the proposed model. Moreover, from the perspective of RMSE, the LSTM model outperforms the other four models with an RMSE of $6.06 \mathrm{mV}$. In short, the presented modeling method based on the LSTM network is more accurate than other traditional modeling methods.

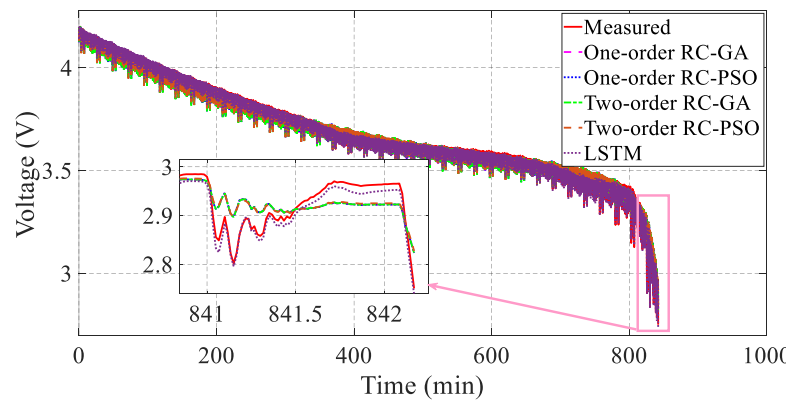

(a)

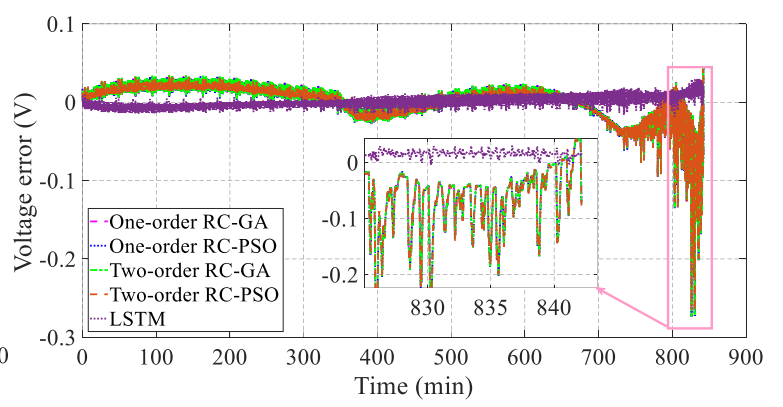

(b)

Fig. 8. Comparison results of different modeling methods. (a) Comparison of voltage. (b) Voltage estimation errors.

TABLE II. EVALUATION RESULTS OF DIFFERENT MODELS.

\begin{tabular}{llll}
\hline \hline Model & AAE $(\mathrm{mV})$ & MAE $(\mathrm{mV})$ & RMSE $(\mathrm{mV})$ \\
\hline First-order RC-GA & 14.53 & 274.01 & 20.46 \\
First-order RC-PSO & 14.52 & 274.00 & 20.46 \\
Second-order RC-GA & 14.52 & 273.30 & 20.42 \\
Second-order RC-PSO & 14.43 & 266.53 & 20.21 \\
LSTM & 4.83 & 29.91 & 6.06 \\
\hline \hline
\end{tabular}




\section{Comparison with Conventional Methods}

To reveal the advantages of the proposed SOC estimation method, we employed two conventional methods, i.e., the first-order ECM and the second-order ECM, to simulate battery electrical performance and applied the PSO algorithm to identify the model parameters. Then, two conventional filters, i.e., unscented Kalman filter (UKF) and SRCKF, are respectively employed to estimate the SOC. Moreover, the UKF and SRCKF are further exploited based on the LSTM model to predict battery SOC. The battery is discharged at room temperature under the FUDS from 100\% SOC. To check the convergence ability of different methods, the initial SOC is erroneously presupposed to $80 \%$.

Fig. 9 and Table III delineate the comparison results of different algorithms and experimentally measured values. The reference profile denotes the real SOC value which is achieved by the Ah integration method. The "ISRCKF" represents the improved SRCKF algorithm. As can be seen, the SRCKF with twoorder RC method leads to the largest error fluctuation of prediction result, and the proposed algorithm can achieve the minimum error, compared with the other estimation results. Several important conclusions can be drawn according to the estimation results based on different commonly used methods. First, the reference SOC of lithium-ion batteries can be tracked accurately in the entire operation range by executing the proposed method. The AAEs of SOC estimation are respectively $0.34 \%, 0.92 \%, 1.52 \%, 3.25 \%, 2.93 \%$, $3.84 \%$ and $1.14 \%$ by the proposed method, SRCKF-LSTM, UKF-LSTM, one-order RC based SRCKF, the first-order RC based UKF, two-order RC based SRCKF and the second-order RC based UKF. Second, thanks to the workmanlike closed-loop feedback, the predicted terminal voltage can converge to the reference value as precisely as the case when the initial SOC is set to $100 \%$. The convergence time for different SOC estimation methods is respectively 2 s, 2 s, 20 s, 100 s, 20 s, 110 s and 6 s, accentuating that the presented method can correct the initial error more quickly than other methods. Last, the SOC estimation results based on the multi-innovation updating technique are more accurate than that of the single error method. The AAE, MAE and RMSE are respectively improved by $62 \%, 64 \%$ and $63 \%$. Hence, the experimental results manifest that the SRCFK along with the multi-innovation technique and LSTM model raises the optimal estimation performance, compared with other commonly used algorithms. 


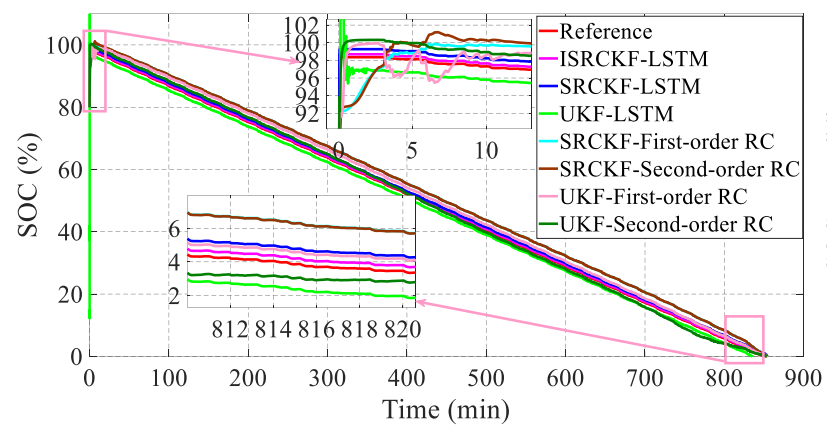

(a)

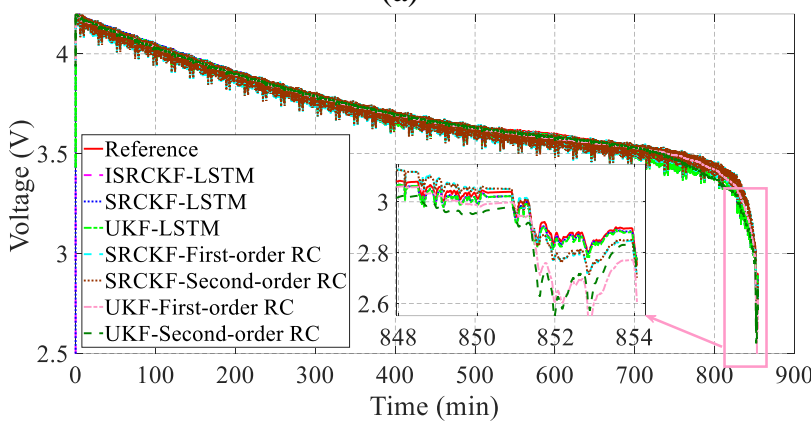

(c)

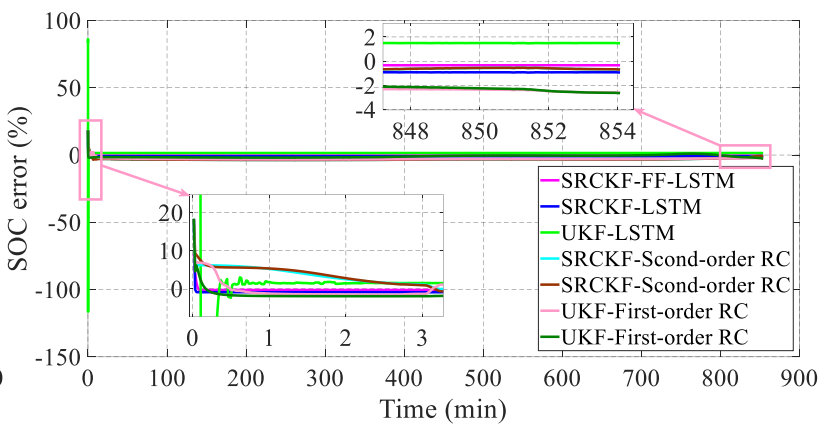

(b)

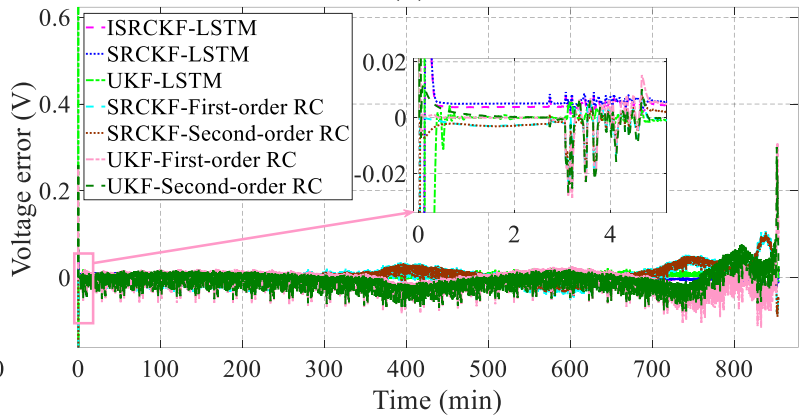

(d)

Fig. 9. SOC evolution with different algorithms and modeling methods. (a) SOC evaluation results. (b) SOC error. (c) Voltage evaluation results. (d) Voltage error.

TABLE III. SUMMARY OF KF-BASED ESTIMATION TECHNIQUES AND BATTERY MODELING METHOD.

\begin{tabular}{|c|c|c|c|c|c|c|c|c|}
\hline \multirow{2}{*}{ Algorithm } & \multirow{2}{*}{ Model } & \multirow{2}{*}{$\begin{array}{l}\text { Convergence } \\
\text { time (s) }\end{array}$} & \multicolumn{3}{|c|}{ SOC (\%) } & \multicolumn{3}{|c|}{ Voltage $(\mathrm{mV})$} \\
\hline & & & AAE & MAE & RMSE & $\mathrm{AAE}$ & MAE & RMSE \\
\hline ISRCKF & & 2 & 0.34 & 0.33 & 0.34 & 4.3 & 23.1 & 20.4 \\
\hline SRCKF & LSTM & 2 & 0.92 & 0.91 & 0.92 & 4.6 & 23.2 & 20.5 \\
\hline UKF & & 20 & 1.52 & 1.79 & 2.07 & 4.3 & 40.9 & 7.3 \\
\hline SRCKF & First- & 100 & 3.25 & 3.33 & 3.30 & 13.4 & 107.8 & 20.7 \\
\hline UKF & order RC & 20 & 2.93 & 2.19 & 2.95 & 13.4 & 311.2 & 20.0 \\
\hline SRCKF & Second- & 110 & 3.26 & 3.84 & 3.29 & 13.3 & 103.6 & 20.5 \\
\hline UKF & order RC & 6 & 1.14 & 0.99 & 1.31 & 13.7 & 300.6 & 21.3 \\
\hline
\end{tabular}

\section{Evaluation Results under Time-Varying Temperature}

The above tests and validations indicate that the proposed LSTM model can effectively simulate the dynamic and static characteristics of lithium-ion batteries, and the improved SRCKF is qualified for SOC estimation at a fixed environment temperature. Nevertheless, the battery is easily perturbed by external environment, and the temperature will change with the operation. Hence, the estimation performance of the proposed method needs to be validated under time-varying temperature conditions. In this study, an operation database containing three experiment schedules, i.e., the dynamic stress test (DST), FUDS test and $\mathrm{CC}-\mathrm{CV}$ charging scheme, records the battery operation with the environmental temperature variation 
from $4{ }^{\circ} \mathrm{C}$ to $53{ }^{\circ} \mathrm{C}$, and is directly employed to evaluate the forecast performance of the presented SOC algorithm.

Fig. 10 (a) and (b) demonstrate the measurement current and temperature, and we can find during the period of 590 minutes to 620 minutes, the temperature curve dropped rapidly from $53{ }^{\circ} \mathrm{C}$ to $9{ }^{\circ} \mathrm{C}$. This process can validate the adaptability of the built estimation algorithm to the time-varying temperature environment. Meanwhile, the test schedule contains high current charge and discharge, followed by the CC-CV charging profile. Fig. 10 (c) and (d) present the SOC prediction results of the proposed algorithm and the LSTM based method. In the LSTM-based SOC estimation algorithm, the input includes current, voltage and temperature of the battery, and the output is the SOC. To make the two models more comparable, the parameters of these models are set to the same values. The selection method of the unit number in the LSTM-based SOC model is similar to that of the LSTM-based voltage model. According to the calculation, the best and worst estimation results appear when the number of units is 100 and 500, and their values are 0.99 and 0.22 , respectively. Thus, 100 hidden units are hired to establish the LSTM network model and estimate SOC.

As can be found from Fig. 10, the LSTM-based SOC estimation can follow the downtrend of SOC, and the MAE and RMSE are $4.5 \%$ and $0.81 \%$ respectively, indicating the prediction SOC is not steady enough. According to the results, it is distinct that large error mainly appears in the lower range of SOC (around 15\%) due to polarization existing at lower SOC. Furthermore, high temperature and rapid variation of temperatures also lead to larger estimation error to some extent. However, the prediction trajectory of the proposed algorithm can match the reference curve with preferable accuracy even in the lower range of SOC. The proposed method can not only track the variation of real SOC for the whole temperature range, but also raise higher estimation precision, compared with that of the LSTM-based method, of which the MAE and RMSE are respectively $1.63 \%$ and $0.31 \%$. By means of comprehensive validation, the practical application potential of the proposed method is justified. 


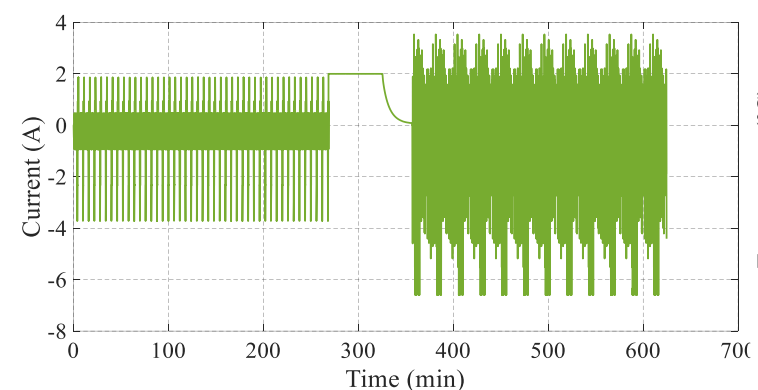

(a)

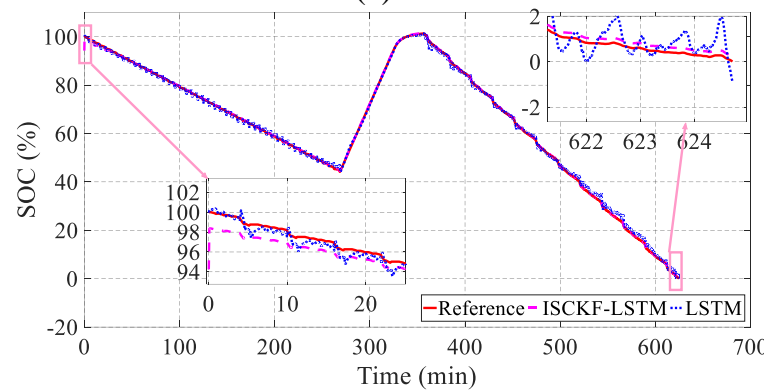

(c)

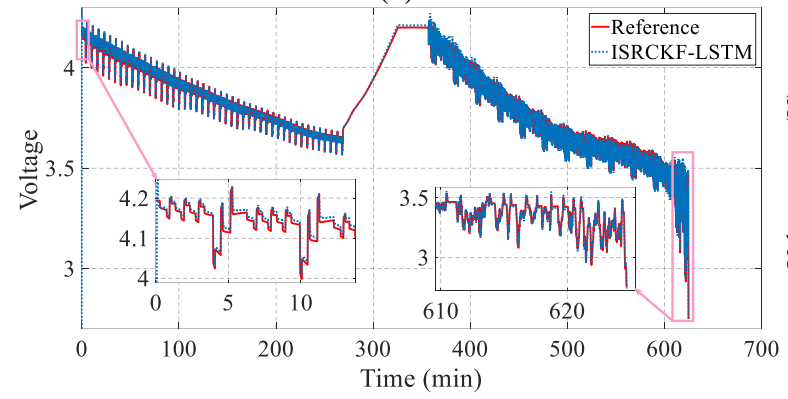

(e)

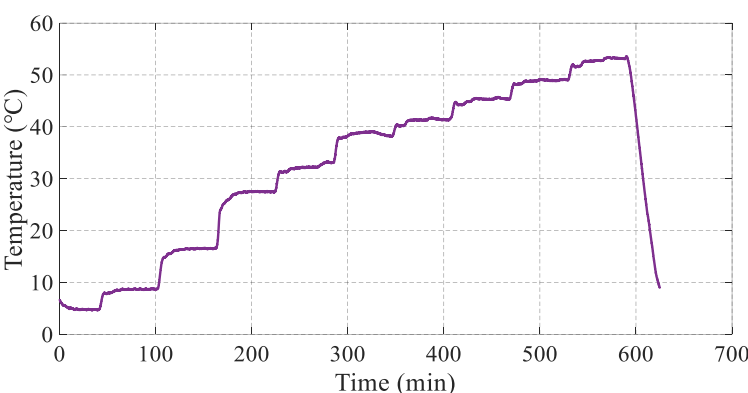

(b)

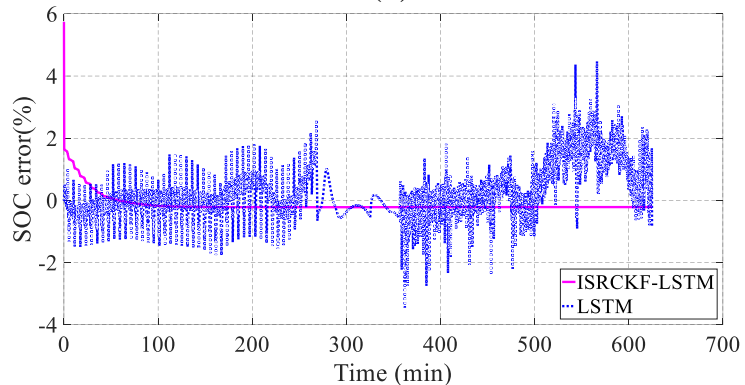

(d)

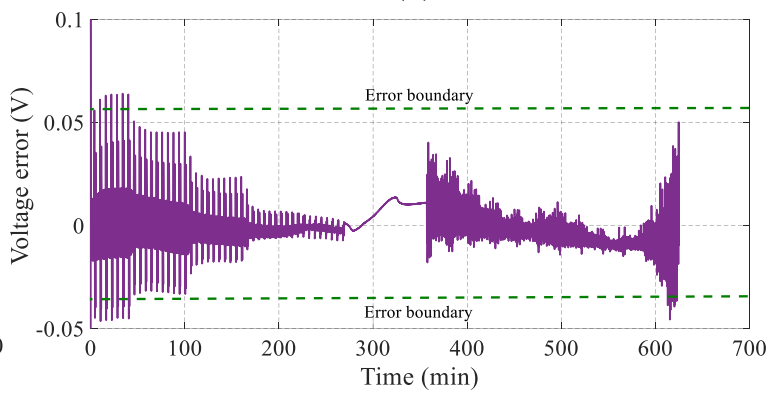

(f)

Fig. 10. Battery cell estimation results under time-vary temperature. (a) Current profiles. (b) Temperature profiles. (c) SOC profiles. (d) SOC error. (e) Voltage profiles. (f) Voltage prediction error.

\section{Performance Evaluation of Battery Pack}

This section uses real data collected by a cloud monitoring platform to verify the battery pack SOC estimation algorithm. Moreover, the data acquisition platform and data processing processes are also elaborated.

\section{A. Data Acquisition}

Cloud system features advantages of strong computational abilities and large storage capacities. Hence, the data from an ES monitoring platform is collected to validate the SOC prediction method for battery pack. The power battery pack consists of 14 modules, each of which is comprised of 6 cells in parallel configuration; and there exist three negative temperature coefficient (NTC) thermistors attached inside the 
battery pack. The data sampling frequency ranges from $0.1 \mathrm{~Hz}$ to $1 \mathrm{~Hz}$. The measured data are further processed and stored on the cloud system.

\section{B. Data Preprocessing}

The original data of current, temperature, pack voltage, velocity and cells' voltage are depicted in Fig. 11. From Fig. 11 (b), we can find that the practical experiment includes rapid acceleration, rapid deceleration, high speed, low speed and halt mode, thus involving all the working models of ESs. The measured temperature variations are plotted in Fig. 11 (c), in which the temperature fluctuates gently, and their effects on battery performance is not significant. Thus, the cell temperature is represented by the mean value of three measured temperatures to simplify the calculation in the estimation process. Fig. 11 (d) shows the cell voltage variation, and we can find that all the cell voltages remain almost the same during operation. The inconsistency among cell voltages can be neglected to some extent. Nevertheless, slight difference of voltage curves appears, as shown in Fig. 11 (d). The maximum and minimum values of voltage appear in cell 4 and cell 13, respectively; and the maximum difference is more than $20 \mathrm{mV}$. Therefore, the estimated SOC of these two cells will be selected to calibrate the battery pack SOC.

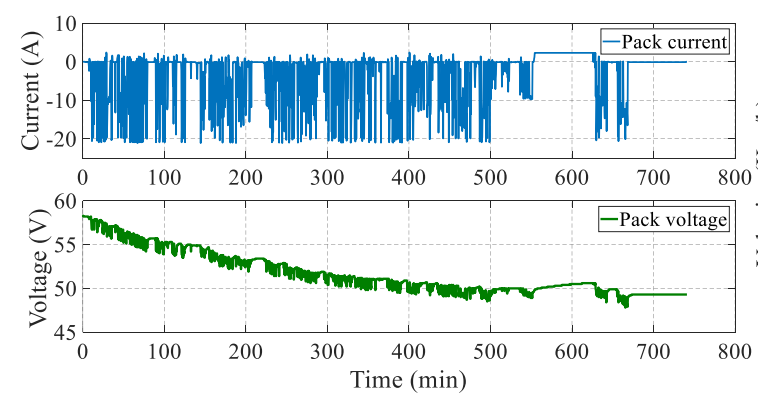

(a)

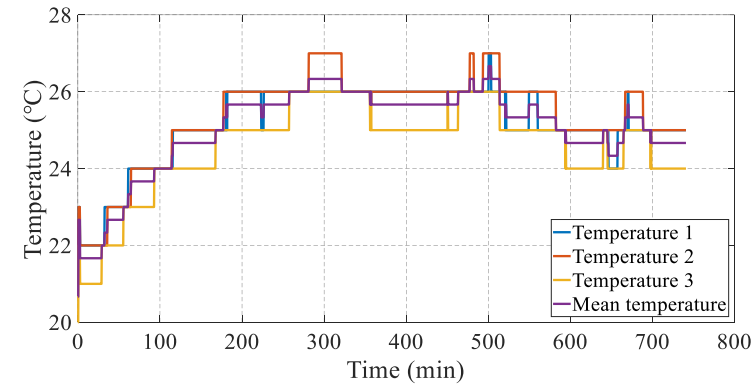

(c)

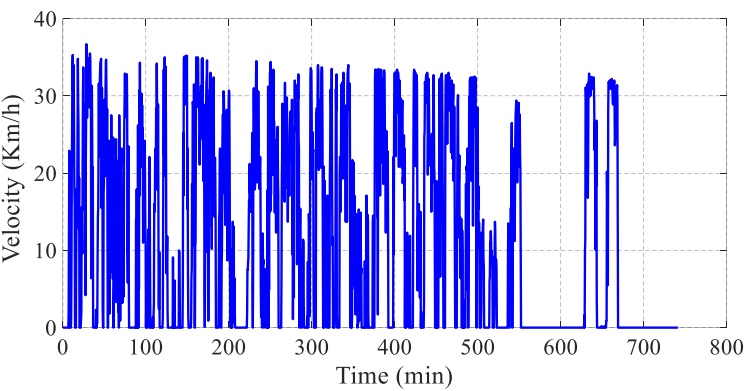

(b)

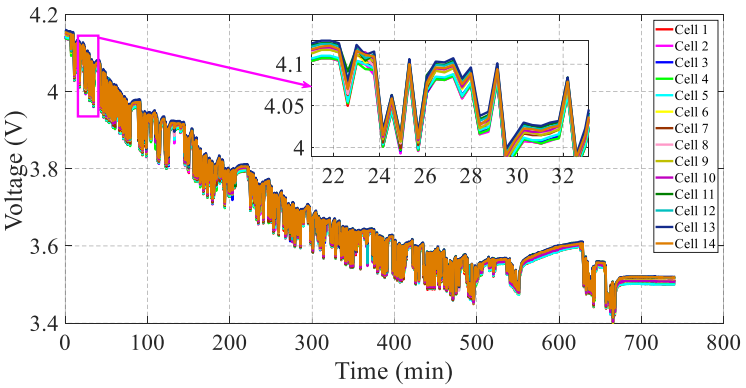

(d)

Fig. 11. ES data curves on November 1, 2019. (a) Battery pack current and voltage. (b) Velocity of ES. (c) Battery pack temperatures. (d) Cell voltages. 


\section{Results and Discussion}

The voltage profile of battery pack under two cycles is drawn in Fig. 12 (a). The voltage, current and temperature in the first cycle are chosen as the training dataset for building the battery model, and the data of the second cycle are considered as the testing dataset. The SOC prediction results and error curves of battery pack based on real-world data are depicted in Fig. 12 (b) and (c), The initial values of minimum SOC and maximum SOC are respectively set to $92 \%$ and $93 \%$. The initial SOC of reference is calculated by checking the standard SOC-OCV look-up table in the equilibrium state. From Fig. 12 (c), it can be observed that the MAE based on the real-world data is less than $2 \%$. Moreover, the battery pack SOC and mean SOC curves are located between the maximum and the minimum of cell SOC. In high SOC range, the battery pack SOC is closer to the maximum value of cell SOC. With the discharge of batteries, the battery pack SOC gradually approaches to the minimum value of cell SOC. When the minimum SOC approximates to 0 , the battery pack SOC also reaches 0 . Fig. 12 (d) exhibits the pack SOC variation when the limitation of SOC is set to $35 \%$. We can find that when the minimum SOC decreases to $35 \%$, the pack SOC concurrently converges to $35 \%$, highlighting the adaptability of the developed pack SOC estimation algorithm and the capability of avoiding over-discharge. Fig. 12 (e) compares the measured and predicted terminal voltage, and Fig. 12 (f) shows that the initial prediction error is up to $100 \mathrm{mV}$, which are generated by the erroneous SOC setting. After the predicted voltage converges to the reference terminal voltages, the MAE of the terminal voltage is only $30 \mathrm{mV}$, excluding a small number of discrete points. Moreover, each step calculation time for battery cell is $2.8 \mathrm{~ms}$, while the sampling interval of battery cell measure is $1 \mathrm{~s}$, which is much longer than the step calculation time for cell SOC estimation. In addition, when all the programs are integrated, each step calculation time when estimating the battery pack SOC is $5.7 \mathrm{~ms}$, still much shorter than the sampling interval. This broadly proves that the pack SOC estimation framework proposed in this paper can meet the requirements of online application, and the feasibility of the proposed estimation method for battery pack SOC is justified. 


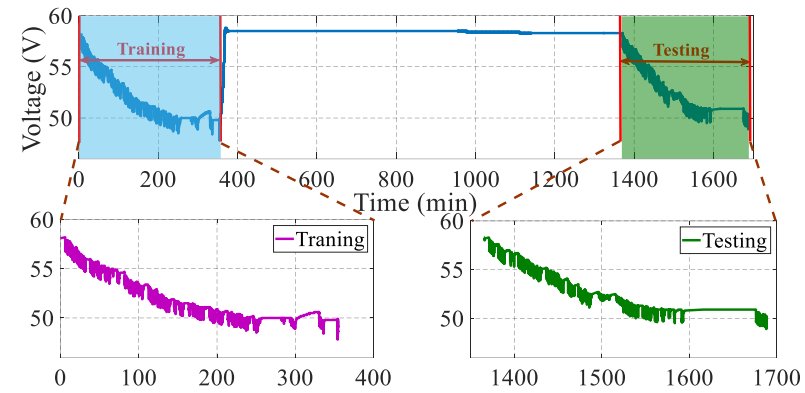

(a)

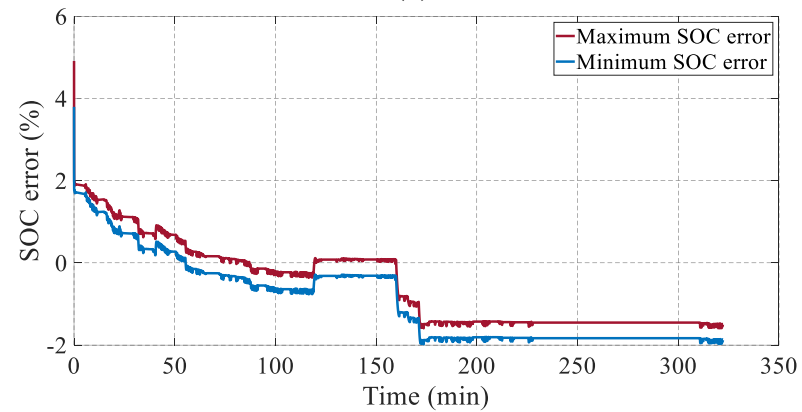

(c)

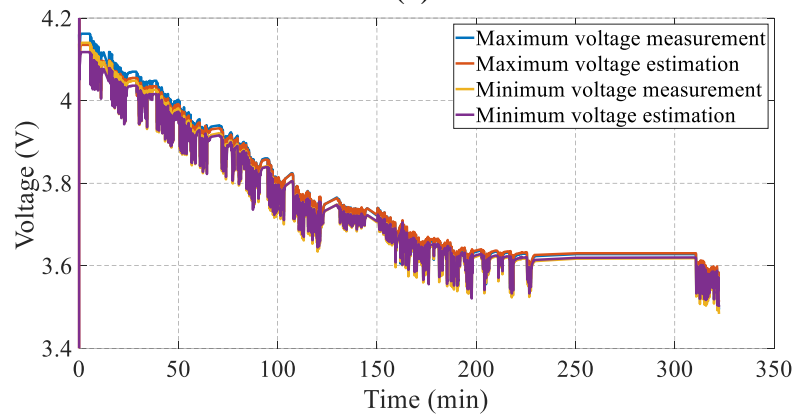

(e)

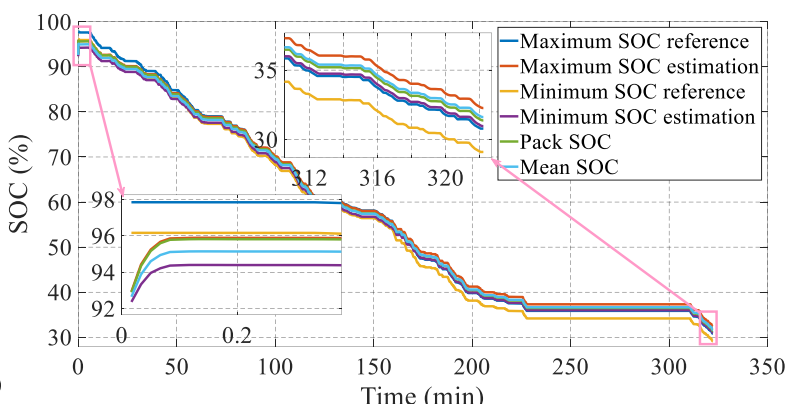

(b)

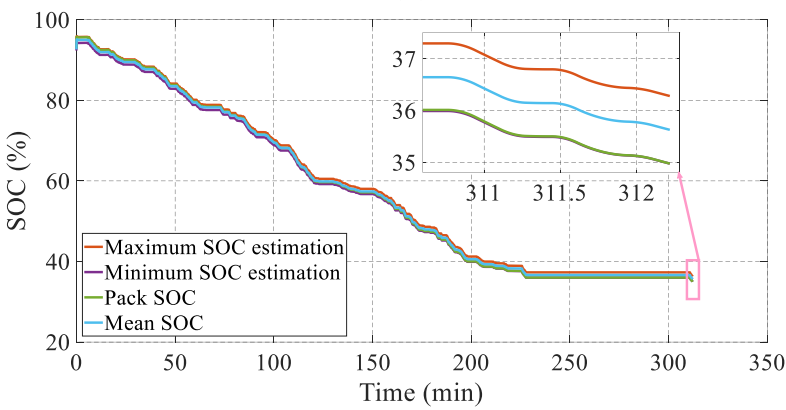

(d)

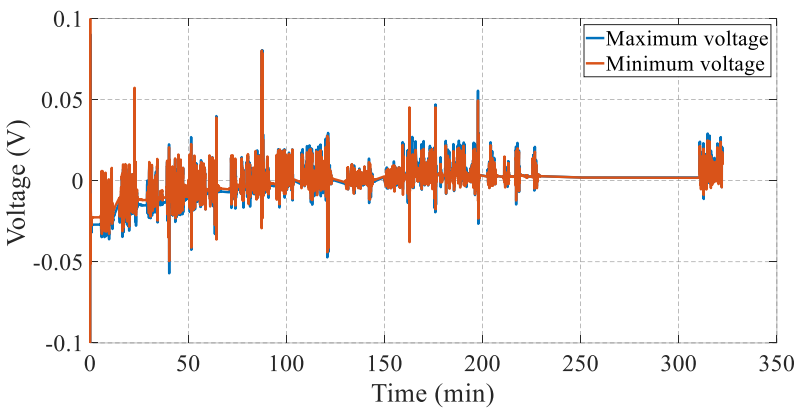

(f)

Fig. 12. Battery pack SOC estimation results. (a) Training and testing datasets. (b) Battery pack SOC estimation results. (c) SOC estimation errors. (d) Battery pack SOC estimation results with 35\% SOC limitation. (e) Voltage estimation results. (f) Voltage estimation errors. Note that "Maximum SOC estimation" and "Minimum SOC estimation" denote the estimated value of maximum and minimum SOC obtained by the ISRCKF-LSTM algorithm. "Pack SOC" is the predicted results of battery pack SOC achieved by the presented method, and "Mean SOC" denotes the calibrated results of battery pack SOC acquired by mean of maximum SOC and minimum SOC.

\section{CONCLUSION}

This paper presents a data-driven battery model based on LSTM RNN to accurately simulate battery characteristics, and a rolling learning method is developed to improve the simulation precision and adapt to environment variation. Then, an improved SRCKF based SOC prediction algorithm is proposed, which is fully substantiated in the case of time-varying temperature environment by comparing with the commonly used SOC estimation algorithms. The experimental results indicate that the cell SOC estimated by the proposed algorithm can quickly converge to the reference value with the RMSE of less than $0.4 \%$ over the 
entire temperature variation range. According to the developed smooth weighing method, the weights and bias of the maximum and minimum SOC for battery cells are dynamically adjusted, and the pack SOC is properly determined. Based on the proposed model and pack SOC estimation method, the MAE of the cell SOC is less than $2 \%$ in ES applications, and the pack SOC can fully consider the thresholds of cell SOC and vary smoothly and reasonably under different operation conditions, verifying its feasibility in practical applications.

Although a reliable battery pack SOC estimation method is proposed in this study, and its model parameters are updated continuously through the rolling learning method, the degradation of battery capacity is not considered in this study. Therefore, the co-estimation of SOC and state of health for lithiumion battery packs in their entire lifespan is the focus of our next step research.

\section{ACKNOWLEDGEMENTS}

This work was supported in part by the National Key R\&D Program of China (No. 2018YFB0104000), in part by the National Natural Science Foundation of China (No. 61763021 and 51775063), and in part by the EU-funded Marie Skłodowska-Curie Individual Fellowships Project under Grant 845102-HOEMEVH2020-MSCA-IF-2018.

\section{REFERENCES}

[1] N. Guo, X. Zhang, Y. Zou, B. Lenzo, and T. Zhang, "A Computationally Efficient Path-Following Control Strategy of Autonomous Electric Vehicles With Yaw Motion Stabilization," IEEE Trans. Transport. Electrific., vol. 6, no. 2, pp. 728-739, 2020, doi: 10.1109/TTE.2020.2993862.

[2] Y. Shang, N. Cui, B. Duan, and C. Zhang, "Analysis and Optimization of Star-Structured Switched-Capacitor Equalizers for Series-Connected Battery Strings," IEEE Trans. Power Electron., vol. PP, no. 99, pp. 1-1, 2017.

[3] X. Shu, G. Li, Y. Zhang, J. Shen, and Y. Liu, "Online diagnosis of state of health for lithium-ion batteries based on short-term charging profiles," J. Power Sources, vol. 471, p. 228478, 2020.

[4] Z. Wei, G. Dong, X. Zhang, J. Pou, Z. Quan, and H. He, "Noise-Immune Model Identification and State of Charge Estimation for Lithium-ion Battery Using Bilinear Parameterization," IEEE Trans. Ind. Electron., pp. 1-1, 2020, doi: 10.1109/TIE.2019.2962429.

[5] Y. Zheng, M. Ouyang, X. Han, L. Lu, and J. Li, "Investigating the error sources of the online state of charge estimation methods for lithium-ion batteries in electric vehicles," J. Power Sources, vol. 377, pp. 161-188, 2018.

[6] X. Hu, F. Feng, K. Liu, L. Zhang, J. Xie, and B. Liu, "State estimation for advanced battery management: Key challenges and future trends," Renew. Sust. Energ. Rev, vol. 114, p. 109334, 2019.

[7] L. Zheng, J. Zhu, D. D.-C. Lu, G. Wang, and T. He, "Incremental capacity analysis and differential voltage analysis based state of charge and capacity estimation for lithium-ion batteries," Energy, vol. 150, pp. 759-769, 2018.

[8] K.-T. Lee, M.-J. Dai, and C.-C. Chuang, "Temperature-compensated model for lithium-ion polymer batteries with extended Kalman filter state-of-charge estimation for an implantable charger," IEEE Trans. Ind. Electron., vol. 65, no. 1, pp. 589-596, 2017. 
[9] M. Ng, J. Zhao, Q. Yan, G. J. Conduit, and Z. W. Seh, "Predicting the state of charge and health of batteries using data-driven machine learning," Nature Machine Intelligence, vol. 2, no. 3, pp. 161-170, 2020.

[10] J. Xu, C. C. Mi, B. Cao, J. Deng, Z. Chen, and S. Li, "The State of Charge Estimation of Lithium-Ion Batteries Based on a Proportional-Integral Observer," IEEE Trans. Veh. Technol., vol. 63, no. 4, pp. 1614-1621, 2014, doi: 10.1109/TVT.2013.2287375.

[11] X. Shu, G. Li, J. Shen, W. Yan, and Y. Liu, "An adaptive fusion estimation algorithm for state of charge of lithium-ion batteries considering wide operating temperature and degradation," J. Power Sources, vol. 462, p. 228132, 2020.

[12] Y. Wang and Z. Chen, "A framework for state-of-charge and remaining discharge time prediction using unscented particle filter," Appl. Energ., vol. 260, p. 114324, 2020.

[13] B. Haus and P. Mercorelli, "Polynomial Augmented Extended Kalman Filter to Estimate the State of Charge of Lithium-Ion Batteries," IEEE Trans. Veh. Technol., vol. 69, no. 2, pp. 1452-1463, 2020, doi: 10.1109/TVT.2019.2959720.

[14] L. Hu, X. Hu, Y. Che, F. Feng, X. Lin, and Z. Zhang, "Reliable state of charge estimation of battery packs using fuzzy adaptive federated filtering," Appl. Energ., vol. 262, p. 114569, 2020.

[15] R. Xiong, H. He, F. Sun, and K. Zhao, "Evaluation on State of Charge Estimation of Batteries With Adaptive Extended Kalman Filter by Experiment Approach," IEEE Trans. Veh. Technol., vol. 62, no. 1, pp. 108-117, 2013, doi: 10.1109/TVT.2012.2222684.

[16] I. Arasaratnam and S. Haykin, "Cubature Kalman Filters," IEEE Trans. Autom. Control, vol. 54, no. 6, pp. 1254-1269, 2009.

[17] P. Shrivastava, T. K. Soon, M. Y. I. B. Idris, and S. Mekhilef, "Overview of model-based online state-of-charge estimation using Kalman filter family for lithium-ion batteries," Renew. Sust. Energ. Rev., vol. 113, p. 109233, 2019.

[18] A. Farmann and D. U. Sauer, "Comparative study of reduced order equivalent circuit models for on-board stateof-available-power prediction of lithium-ion batteries in electric vehicles," Appl. Energ., vol. 225, pp. 1102$1122,2018$.

[19] M. S. E. Din, A. A. Hussein, and M. F. Abdel-Hafez, "Improved Battery SOC Estimation Accuracy Using a Modified UKF With an Adaptive Cell Model Under Real EV Operating Conditions," IEEE Trans. Transport. Electrific., vol. 4, no. 2, pp. 408-417, 2018, doi: 10.1109/TTE.2018.2802043.

[20] X. Lai, S. Wang, S. Ma, J. Xie, and Y. Zheng, "Parameter sensitivity analysis and simplification of equivalent circuit model for the state of charge of lithium-ion batteries," Electrochim. Acta, vol. 330, p. 135239, 2020.

[21] X. Shu, G. Li, J. Shen, Z. Lei, Z. Chen, and Y. Liu, "A uniform estimation framework for state of health of lithium-ion batteries considering feature extraction and parameters optimization," Energy, vol. 204, p. 117957, 2020.

[22] X. Shu, G. Li, J. Shen, Z. Lei, Z. Chen, and Y. Liu, "An adaptive multi-state estimation algorithm for lithiumion batteries incorporating temperature compensation," Energy, vol. 207, p. 118262, 2020.

[23] J. Tian, R. Xiong, and Q. Yu, "Fractional-Order Model-Based Incremental Capacity Analysis for Degradation State Recognition of Lithium-Ion Batteries," IEEE Trans. Ind. Electron., vol. 66, no. 2, pp. 1576-1584, 2019, doi: 10.1109/TIE.2018.2798606.

[24] Z. Wei, J. Zhao, C. Zou, T. M. Lim, and K. J. Tseng, "Comparative study of methods for integrated model identification and state of charge estimation of lithium-ion battery," J. Power Sources, vol. 402, no. OCT.31, pp. 189-197, 2018.

[25] G. O. Sahinoglu, M. Pajovic, Z. Sahinoglu, Y. Wang, P. V. Orlik, and T. Wada, "Battery State-of-Charge Estimation Based on Regular/Recurrent Gaussian Process Regression," IEEE Trans. Ind. Electron., vol. 65, no. 5, pp. 4311-4321, 2018, doi: 10.1109/TIE.2017.2764869.

[26] J. Hong, Z. Wang, W. Chen, and Y. Yao, "Synchronous multi-parameter prediction of battery systems on electric vehicles using long short-term memory networks," Appl. Energ., vol. 254, p. 113648, 2019.

[27] Y. Wu, Q. Xue, J. Shen, Z. Lei, Z. Chen, and Y. Liu, "State of Health Estimation for Lithium-Ion Batteries Based on Healthy Features and Long Short-Term Memory," IEEE Access, vol. 8, pp. 28533-28547, 2020, doi: 10.1109/ACCESS.2020.2972344.

[28] F. Feng, X. Hu, L. Hu, F. Hu, Y. Li, and L. Zhang, "Propagation mechanisms and diagnosis of parameter inconsistency within Li-Ion battery packs," Renew. Sust. Energ. Rev, vol. 112, no. SEP., pp. 102-113, 2019.

[29] C. Yang, X. Wang, Q. Fang, H. Dai, and X. Wei, "An online SOC and capacity estimation method for aged lithium-ion battery pack considering cell inconsistency," J. Energy Storage, vol. 29, p. 101250, 2020. 
[30] Y. Zheng, W. Gao, M. Ouyang, L. Lu, L. Zhou, and X. Han, "State-of-charge inconsistency estimation of lithium-ion battery pack using mean-difference model and extended Kalman filter," J. Power Sources, vol. 383, pp. 50-58, 2018.

[31] J. Hong, Z. Wang, and Y. Yao, "Fault prognosis of battery system based on accurate voltage abnormity prognosis using long short-term memory neural networks," Appl. Energ., vol. 251, p. 113381, 2019/10/01/2019, doi: https://doi.org/10.1016/j.apenergy.2019.113381.

[32] X. Cui, Z. He, E. Li, A. Cheng, M. Luo, and Y. Guo, "State-of-charge estimation of power lithium-ion batteries based on an embedded micro control unit using a square root cubature Kalman filter at various ambient temperatures," Int. J. Energ. Res., vol. 43, no. 8, pp. 3561-3577, 2019.

[33] S. Zhang, X. Guo, and X. Zhang, "Multi-objective decision analysis for data-driven based estimation of battery states: A case study of remaining useful life estimation," Int. J. Hydrogen. Energ., vol. 45, no. 27, pp. 1415614173, 2020/05/18/ 2020, doi: https://doi.org/10.1016/j.ijhydene.2020.03.100. 